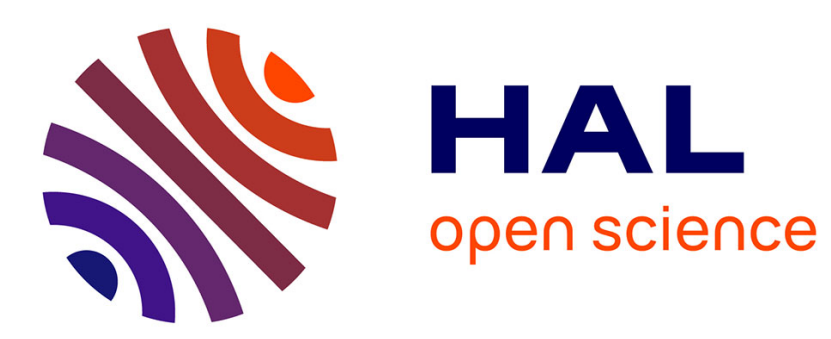

\title{
Improving quantum-noise reduction with spatially multimode squeezed light
}

\author{
Luigi A. Lugiato, Philippe Grangier
}

\section{To cite this version:}

Luigi A. Lugiato, Philippe Grangier. Improving quantum-noise reduction with spatially multimode squeezed light. Journal of the Optical Society of America B, 1997, 14 (2), pp.225-231. 10.1364/JOSAB.14.000225 . hal-00871013

\section{HAL Id: hal-00871013 \\ https://hal-iogs.archives-ouvertes.fr/hal-00871013}

Submitted on 8 Oct 2013

HAL is a multi-disciplinary open access archive for the deposit and dissemination of scientific research documents, whether they are published or not. The documents may come from teaching and research institutions in France or abroad, or from public or private research centers.
L'archive ouverte pluridisciplinaire HAL, est destinée au dépôt et à la diffusion de documents scientifiques de niveau recherche, publiés ou non, émanant des établissements d'enseignement et de recherche français ou étrangers, des laboratoires publics ou privés. 


\title{
Improving quantum-noise reduction with spatially multimode squeezed light
}

\author{
L. A. Lugiato \\ Dipartimento di Fisica dell'Universita di Milano, Via Celoria 16, 20133 Milano, Italy \\ Ph. Grangier \\ Institut d'Optique Théorique et Appliquée, Bâtiment 503, B.P. 147, F91403 Orsay, France
}

Received November 8, 1995; revised manuscript received May 21, 1996

\begin{abstract}
We consider the squeezed vacuum state emitted by a degenerate optical parametric oscillator below threshold. We show, by using a confocal cavity and an appropriate matching lens, that the observed level of squeezing can become largely independent of the spatial intensity configuration of the local-oscillator field. (C) 1997 Optical Society of America [S0740-3224(97)01002-3]

PACS number(s): $42.50 . \mathrm{Dv}, 42.65 . \mathrm{Ky}$
\end{abstract}

\section{INTRODUCTION}

Although theoretical studies on squeezed states started more than fifteen years ago, ${ }^{1}$ the majority of these investigations dealt only with temporal/frequency aspects, neglecting all features related to space. Until recently, the exceptions were only few, and in this connection we would like to mention the pioneering works of Refs. 2-8.

In particular, in the travelling wave case, the analysis of Ref. 5 showed that, in the detection of the squeezed beam emitted by an optical parametric amplifier, the observed level of squeezing can be substantially degraded by the poor matching between the transverse configurations of the deamplified signal beam and of the Gaussian local-oscillator (LO) field. An answer to this difficulty was proposed and implemented recently by matching a nonGaussian shape of the LO to the distorted squeezed vacuum. ${ }^{6}$

For squeezed fields emitted by nonlinear cavities, two recent papers ${ }^{7,8}$ analyze carefully the dependence of the observed level of squeezing on the spatial configuration of the LO field, with the aim of providing an operational description of the spatial structure of squeezed states. This approach was then applied to the case of the degenerate optical parametric oscillator (OPO) below threshold. ${ }^{7,8}$

We will use here an approach inspired by these papers to answer the following question: Is it possible, under appropriate conditions and to a significant degree, to ensure that the observed level of squeezing becomes independent of the spatial configuration of the LO field? By following the approach developed in Refs. 7 and 8, we show in this paper that the answer is positive in the case of the squeezed vacuum field emitted by a degenerate OPO below threshold. Clearly, this possibility could be quite convenient in practice, because it allows one to avoid or at least simplify delicate mode-matching techniques. The key point in our analysis is the use of a confocal cavity, for which all the Gauss-Laguerre cavity modes of even order are frequency degenerate. As it will be explained in Section 4, the mode matching can then be done with a lens that does not depend directly on the LO beam shape and size.

In Section 2 we present again the quantum model of the OPO below threshold, including diffraction effects, and we express the model in terms of Gauss-Laguerre modes. Section 3 reviews the general definition of the spectrum of squeezing, including spatial aspects, ${ }^{7,8}$ and, in the case of the degenerate OPO below threshold, expresses the spectrum in terms of single mode contributions. The main part of the analysis is contained in Section 4, in which we show that the observed level of squeezing is partially independent of the intensity configuration in the LO field. In Section 5 we compare the technique used here with standard mode matching. Section 6 summarizes the results obtained and contains several considerations and comments.

\section{QUANTUM MODEL FOR THE OPTICAL PARAMETRIC OSCILLATOR BELOW THRESHOLD}

For our purpose, we will have to consider a modedegenerate cavity that we will simply assume here to be confocal, i.e., made of two spherical mirrors separated by a distance equal to their radius of curvature. We will assume that the cavity contains a $\chi^{(2)}$ crystal (Fig. 1), which converts a pump field of frequency $2 \omega_{S}$ into a signal field of frequency $\omega_{S}$ and vice versa. The crystal is located at the center of the cavity and is much shorter than the Rayleigh length that characterizes diffraction (or equivalently, much shorter than the cavity length). The cavity center will be taken as the reference plane $z=0$, and the cavity beam waist will be denoted $w_{C}$. Because of its cylindrical symmetry, the cavity supports a complete set of Gauss-Laguerre modes ${ }^{9}$ that have the following transverse configuration in the vicinity of the cavity center: 


$$
\begin{aligned}
A_{p 0}(r, \varphi, z=0)= & \frac{2}{w_{C} \sqrt{2 \pi}} L_{p}^{0}\left(\frac{2 r^{2}}{w_{C}^{2}}\right) \exp \left(-\frac{r^{2}}{w_{C}^{2}}\right), \\
A_{p \ell i}(r, \varphi, 0)= & \frac{2}{w_{C} \sqrt{\pi}}\left(\frac{2 r^{2}}{w_{C}^{2}}\right)^{\ell / 2}\left(\frac{p !}{(p+\ell) !}\right)^{1 / 2} L_{p}^{\ell}\left(\frac{2 r^{2}}{w_{C}^{2}}\right) \\
& \times \exp \left(-\frac{r^{2}}{w_{C}^{2}}\right) \begin{cases}\cos (\ell \varphi) & \text { for } i=1 \\
\sin (\ell \varphi) & \text { for } i=2,\end{cases}
\end{aligned}
$$

where $p, \ell=0,1,2, \ldots$ and $p$ (respectively, $\ell$ ) is the radial (respectively, angular) index; $r$ denotes the radial coordinate $\sqrt{x^{2}+y^{2}}$, and $L_{p}^{\ell}$ are Laguerre polynomials of the indicated argument. All the modes with $\ell$ even have the same frequency; the same is true for the modes with $\ell$ odd; the frequency separation between the two groups of modes is equal to one half the free spectral range. Since the crystal is close to the beam waist and much shorter than the Rayleigh length, the configuration of the GaussLaguerre modes along the crystal is still given by Eqs. (1) and (2). Moreover, we assume the validity of the paraxial and the slowly varying approximations and of the mean field-limit conditions so that the envelopes of all the fields are uniform along the crystal.

In the OPO below threshold one can neglect the depletion of the pump field and treat the pump field as a classical quantity, described by a coherent, stationary field $E_{p}$ of frequency $2 \omega_{S}$. An important assumption will be that the input pump field has an axial plane-wave configuration (i.e., a uniform field distribution) in correspondence to the crystal. The simplest way to implement this condition is to assume that the pump-beam waist is much larger than the cavity beam waist at the crystal location. In practice, the pump field should not be reflected by the cavity mirrors and should simply travel once through the crystal. For the signal beam we assume that one mirror has a high reflectivity and that the other one is completely reflecting; hence the cavity has only one inputoutput port at the signal wavelength. Finally, we assume that the frequency $\omega_{S}$ of the signal field is close to resonance with a frequency-degenerate set of GaussLaguerre modes with $\ell$ even.

The quantum model for the OPO below threshold can be expressed in terms of an appropriate master equation that governs the time evolution of the intracavity signal field $A_{C}(\mathbf{x})$ at cavity center, which obeys the commutation rule:

$$
\left[A_{C}(\mathbf{x}), A_{C}^{+}\left(\mathbf{x}^{\prime}\right)\right]=\delta\left(\mathbf{x}-\mathbf{x}^{\prime}\right)
$$

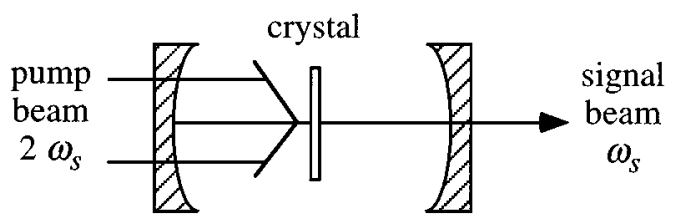

Fig. 1. OPO using a confocal cavity. At the signal frequency $\omega_{S}$, the input-output mirror has a high reflectivity, whereas the other spherical mirror has $100 \%$ reflectivity. Both mirrors transmit the pump field $E_{p}$ of frequency $2 \omega_{S}$. where $\mathbf{x}=(x, y)$, and $x, y$ are the Cartesian coordinates in the transverse plane. The field $A_{C}(\mathbf{x})$ can be expanded on the basis of the Gauss-Laguerre modes:

$$
A_{C}(\mathbf{x})=\sum_{p \ell i} A_{p \ell i}(r, \varphi, 0) a_{p \ell i}
$$

where the mode operators $a_{p / i}$ obey the commutation rule,

$$
\left[a_{p \ell i}, a_{p^{\prime} \ell^{\prime} i^{\prime}}^{+}\right]=\delta_{p, p^{\prime}} \delta_{\ell, \ell^{\prime}} \delta_{i, i^{\prime}} .
$$

If we indicate by $\rho$ the density operator of the intracavity signal field in the interaction picture, the master equation reads ${ }^{7,8}$

$$
\frac{\mathrm{d} \rho}{\mathrm{d} t}=\frac{1}{i \hbar}[H, \rho]+\sum_{p \ell i}{ }^{\prime} \Lambda_{p \ell i} \rho .
$$

The prime in $\Sigma_{p / i}^{\prime}$ means that in the summation we include only the set of degenerate Gauss-Laguerre modes with $\ell$ even, which are quasi-resonant with the signal field frequency $\omega_{s}$, and call $\omega_{c}$ their frequency. As a matter of fact, practically all of the other cavity modes do not interact with the nonlinear material and simply remain in the vacuum state.

The Liouvillian terms

$$
\Lambda_{p / i}=\gamma\left[2 a_{p \ell i} \rho a_{p / i}^{+}-\rho a_{p / i}^{+} a_{p / i}-a_{p / i}^{+} a_{p / i} \rho\right]
$$

describe the escape of signal photons from the cavity, which occurs with the rate $\gamma$. The Hamiltonian $H$ is given by:

$$
H=H_{\mathrm{FREE}}+H_{\mathrm{INT}},
$$

where $H_{\text {FREE }}$ describes the free evolution of the cavity modes:

$$
H_{\mathrm{FREE}}=\hbar\left(\omega_{c}-\omega_{s}\right) \sum_{p / i}{ }^{\prime} a_{p / i}^{+} a_{p / i}
$$

The interaction Hamiltonian $H_{\mathrm{INT}}$ is given by

$$
\begin{aligned}
H_{\mathrm{INT}} & =i \hbar \frac{A_{p}}{2} \int \mathrm{d}^{2} \mathbf{x}\left\{\left[A_{C}^{+}(\mathbf{x})\right]^{2}-A_{C}^{2}(\mathbf{x})\right\} \\
& =i \hbar \frac{A_{p}}{2} \sum_{p \ell i}{ }^{\prime}\left[\left(a_{p / i}^{+}\right)^{2}-a_{p / i}^{2}\right],
\end{aligned}
$$

where the $C$ number $A_{p}$ is proportional to the $\chi^{(2)}$ coefficient of the material and to the amplitude $E_{p}$ of the pump field, which as before is assumed to be independent of $\mathbf{x}$, and is therefore taken out of the integral. The last expression in Eq. (10) arises from the orthonormality and the reality of the modes (1) and (2). As we said, only the modes with even $\ell$ are involved in the interaction, because the other modes are too far from resonance with respect to the signal frequency $\omega_{s}$.

As we can see, from Eqs. (7)-(10), master Eq. (6) describes the evolution of an infinite set of uncoupled, single-mode optical parametric oscillators with the same frequency, corresponding to the even Gauss-Laguerre modes. 


\section{BALANCED HOMODYNE DETECTION AND SPECTRUM OF SQUEEZING}

The well-known balanced homodyne detection scheme for squeezed states is illustrated in Fig. 2. In the propagation from the cavity center $(z=0)$ to the beam splitter $\left(z=z_{\mathrm{LO}}\right.$ ), the configuration of the Gauss-Laguerre modes changes owing to diffraction and to possible matching lenses, which will be discussed later in the paper. Let us indicate as $A_{p \ell i}(r, \varphi, z)$ the configuration of the Gauss-Laguerre modes at a generic distance $z$ from the cavity center [the explicit expression of $A_{p / i}(r, \varphi, z)$ is given in Appendix A]. Because the transformation of the field from the plane $z=0$ to the plane $z$ is unitary, the functions $A_{p / i}(r, \varphi, z)$ obey in any plane $z$ the orthonormality relations:

$$
\begin{aligned}
\int_{0}^{\infty} r \mathrm{~d} r \int_{0}^{2 \pi} \mathrm{d} \varphi A_{p \ell i}^{*}(r, \varphi, z) A_{p^{\prime} \ell^{\prime} i^{\prime}} & (r, \varphi, z) \\
& =\delta_{p, p^{\prime}} \delta_{\ell, \ell^{\prime}} \delta_{i, i^{\prime}} .
\end{aligned}
$$

In addition, because the transformation is linear, the field $A_{C}(\mathbf{x})=A(\mathbf{x}, z=0)$ given by Eq. (4) at the cavity center will become, at the position $z$,

$$
A(\mathbf{x}, z)=\sum_{p \ell i} A_{p / i}(r, \varphi, z) a_{p / i}
$$

For describing the homodyne detection it is convenient to define the quantum input and output fields $A_{\text {in }}(\mathbf{x}, t)$ and $A_{\text {out }}(\mathbf{x}, t)$ at the beam-splitter location $z=z_{\mathrm{LO}}$. Because of the output mirror of the cavity, these fields are related by

$$
A_{\text {out }}(\mathbf{x}, t)=\sqrt{2 \gamma} A\left(\mathbf{x}, z_{\mathrm{LO}}\right)-A_{\text {in }}(\mathbf{x}, t),
$$

where all fields are evaluated at $z=z_{\mathrm{LO}}$.

The beam splitter $\mathrm{M}$ combines the quantum-field envelope operator $A_{\text {out }}(\mathbf{x}, t)$ and the local-oscillator field that lies in a classical stationary coherent state $\alpha_{L}(\mathbf{x})\left[\left|\alpha_{L}(\mathbf{x})\right|^{2}\right.$ is expressed in inverse square centimeters per inverse second]. The operator $A_{\text {out }}$ obeys the commutation rule:

$$
\left[A_{\text {out }}(\mathbf{x}, t), A_{\text {out }}^{+}\left(\mathbf{x}^{\prime}, t^{\prime}\right)\right]=\delta\left(\mathbf{x}-\mathbf{x}^{\prime}\right) \delta\left(t-t^{\prime}\right) .
$$

Assuming that the transmissivity and the reflectivity coefficients of the mirror M (Fig. 2) are given by $t=r$ $=1 / \sqrt{2}$, the fields $B_{1}$ and $B_{2}$ beyond the mirrors are given by

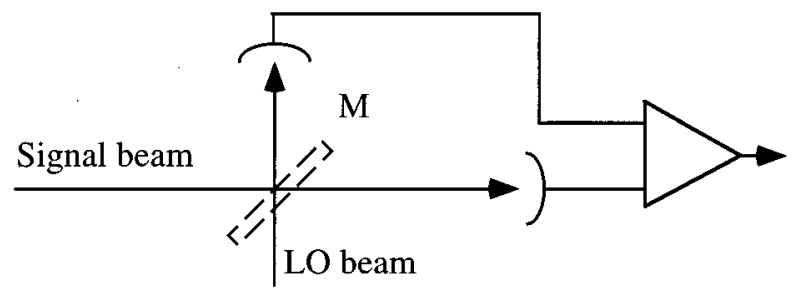

Fig. 2. Balanced homodyne detection scheme. The mirror $M$ has transmission and reflection coefficients $t=1 /(2)^{1 / 2}$ and $r$ $=1 /(2)^{1 / 2}$ for the field amplitude.

$$
\begin{aligned}
& B_{1}(\mathbf{x}, t)=\frac{1}{\sqrt{2}}\left[A_{\text {out }}(\mathbf{x}, t)+\alpha_{L}(\mathbf{x})\right], \\
& B_{2}(\mathbf{x}, t)=\frac{1}{\sqrt{2}}\left[A_{\text {out }}(\mathbf{x}, t)-\alpha_{L}(\mathbf{x})\right],
\end{aligned}
$$

One measures the difference between the total powers of the two beams $B_{1}$ and $B_{2}$, which is given by

$$
\begin{array}{r}
\int \mathrm{d}^{2} \mathbf{x}\left[B_{1}^{+}(\mathbf{x}, t) B_{1}(\mathbf{x}, t)-B_{2}^{+}(\mathbf{x}, t) B_{2}(\mathbf{x}, t)\right] \\
=N^{-1 / 2} E_{A}^{(\text {out })}(t),
\end{array}
$$

where

$$
\begin{aligned}
N= & \int \mathrm{d}^{2} \mathbf{x}\left|\alpha_{L}(\mathbf{x})\right|^{2}, \\
E_{A}^{(\text {out })}(t)= & \frac{1}{N^{1 / 2}} \int \mathrm{d}^{2} \mathbf{x}\left[\alpha_{L}^{*}(\mathbf{x}) A_{\text {out }}(\mathbf{x}, t)\right. \\
& \left.+\alpha_{L}(\mathbf{x}) A_{\text {out }}^{+}(\mathbf{x}, t)\right] .
\end{aligned}
$$

The fluctuations around steady state are described by the spectrum

$$
V(\omega)=\int_{-\infty}^{+\infty} \mathrm{d} t \exp (-i \omega t)\left\langle\delta E_{A}^{(\text {out })}(t) \delta E_{A}^{(\text {out })}(0)\right\rangle,
$$

where

$$
\delta E_{A}(t)=E_{A}^{(\text {out })}(t)-\left\langle E_{A}^{(\text {out })}\right\rangle
$$

and $\left\langle E_{A}^{(\text {out })}\right\rangle$ is the stationary mean value. By using the definitions given above, one easily obtains

$$
V(\omega)=1+S(\omega)
$$

with

$$
S(\omega)=\int_{-\infty}^{+\infty} \mathrm{d} t \exp (-i \omega t)\left\langle: \delta E_{A}^{\text {(out) }}(t) \delta E_{A}^{(\text {out })}(0):\right\rangle,
$$

where :: indicates normal and time ordering. The first term in Eq. (21) represents the shot noise level, which is normalized to 1 . One has $S(\omega) \geqslant-1$, and $S(\omega)=-1$ means that there is complete suppression of quantum noise at frequency $\omega$ in the observable $E_{A}^{\text {(out) }}$.

Inserting Eq. (13) into Eq. (22), and taking into account that $A_{\text {in }}$ (in the vacuum state) does not contribute to the normal and time-ordered product, we obtain

$$
S(\omega)=2 \gamma \int_{-\infty}^{+\infty} \mathrm{d} t \exp (-i \omega t)\left\langle: \delta E_{A}(t) \delta E_{A}(0):\right\rangle,
$$

where

$$
\begin{aligned}
E_{A}(t)= & \frac{1}{N^{1 / 2}} \int \mathrm{d}^{2} \mathbf{x}\left[\alpha_{L}(\mathbf{x}) A^{+}\left(\mathbf{x}, z_{\mathrm{LO}}\right)\right. \\
& \left.+\alpha_{L}^{*}(\mathbf{x}) A\left(\mathbf{x}, z_{\mathrm{LO}}\right)\right] .
\end{aligned}
$$

By inserting expansion (12) into Eqs. (23) and (24) and taking into account that the different modes are uncorrelated, one obtains easily that the spectrum can be expressed in the following form: 


$$
S(\omega)=\sum_{p \ell i}^{\prime} \rho_{p \ell i}^{2} S_{p \ell i}(\omega)
$$

where

$$
S_{p \ell i}(\omega)=2 \gamma \int_{-\infty}^{+\infty} \mathrm{d} t \exp (-i \omega t)\left\langle: \delta b_{p \ell i}(t) \delta b_{p \ell i}(0):\right\rangle
$$

and we have set

$$
b_{p / i}=a_{p / i}^{+} \exp \left(i \varphi_{p / i}\right)+a_{p / i} \exp \left(-i \varphi_{p / i}\right)
$$

and $\left(\rho_{p / i}\right.$ is a real number)

$$
\begin{aligned}
\rho_{p / i} \exp \left(-i \varphi_{p / i}\right)= & \frac{1}{N^{1 / 2}} \int_{0}^{\infty} r \mathrm{~d} r \int_{0}^{2 \pi} \mathrm{d} \varphi \alpha_{L}^{*}(r, \varphi) \\
& \times A_{p / i}\left(r, \varphi, z_{\mathrm{LO}}\right) .
\end{aligned}
$$

The sum in Eq. (25) has again been restricted to the modes with $\ell$ even because the odd modes are too far from resonance and do not feel the interaction with the nonlinear material. The expression of the single-mode spectra [Eq. (26)] is well known in the literature ${ }^{10,11}$ and is given by

$$
\begin{aligned}
S_{p \ell i}(\omega) & \\
= & 4 \bar{A}_{p} \\
& \times \frac{\left\{2 \bar{A}_{p}-\operatorname{Re}\left[\left(1+\omega^{2}-\Delta^{2}+\bar{A}_{p}^{2}-2 i \Delta\right) \exp \left(-2 i \varphi_{p / i}\right)\right]\right\}}{\left(1+\Delta^{2}-\bar{A}_{p}^{2}-\bar{\omega}^{2}\right)^{2}+4 \bar{\omega}^{2}},
\end{aligned}
$$

where $\bar{A}_{p}=A_{p} / \gamma, \Delta=\left(\omega_{c}-\omega_{s}\right) / \gamma$, and $\bar{\omega}=\omega / \gamma$.

We obtained therefore the very intuitive result that the total squeezing [Eq. (25)] is a weighted sum of singlemode spectra (29), where the weighting factor involves the square modulus of scalar product (28) between the amplitude of the LO beam at a given plane and each mode propagated up to the same plane. The phase $\varphi_{p / i}$ of the scalar product determines whether the noise is reduced or increased, as shown by Eq. (29). In Section 4 we will present simple conditions so that $\varphi_{p / i}$ does depend on the mode and so that maximum noise reduction can be obtained.

\section{OBSERVED SQUEEZING AND SPATIAL SHAPE OF THE LOCAL-OSCILLATOR FIELD}

In the previous section all fields were evaluated at the beam-splitter location. However, in the following it will be necessary to consider other positions for both the LO and the signal fields. We will indicate by $\alpha(\mathbf{x}, z)$ the configuration of the $\mathrm{LO}$ at a generic position $z$, with $\alpha\left(\mathbf{x}, z_{\mathrm{LO}}\right)=\alpha_{L}(\mathbf{x})$, as defined previously. Let us now formulate the two following assumptions concerning the local oscillator:

(i) The LO field has nonvanishing projection $\rho_{p / i}$ [see Eq. (28)] only for $\ell$ even. This is equivalent to the requirement that the LO have the symmetry $\alpha_{L}(r, \varphi+\pi)$ $=\alpha_{L}(r, \varphi)$. (ii) There is a plane $P$ for which the phase of the LO is constant modulo $\pi$ but where the spatial distribution of its intensity remains arbitrary. Hence we can write

$$
\alpha\left(\mathbf{x}, z_{P}\right)=\rho_{P}(\mathbf{x}) \exp \left(i \varphi_{P}\right),
$$

where $\varphi_{P}$ is constant and $\rho_{P}(\mathbf{x})$ is real but not necessarily positive and fulfills condition (i). For example, $\rho_{P}(\mathbf{x})$ could correspond to arbitrary Gauss-Laguerre mode (1) multiplied by an arbitrary phase factor $\exp \left(i \varphi_{P}\right)$, but we note that cylindrical symmetry is not required (an on-axis elliptical beam will work equally well).

Let us call $d$ the distance between the plane $P$ (on the axis $z$, see Fig. 3 ) and the plane $C$. We locate, halfway between planes $P$ and $C$, a lens of focal length $f=d / 2$. This lens will transform a Gaussian beam of beam waist $w_{c}$ in plane $C$ into a Gaussian beam of beam waist $w_{P}$ in plane $P$, in such a way that the Rayleigh lengths $r_{c}$ $=\pi w_{C}^{2} / \lambda$ and $r_{P}=\pi w_{P}^{2} / \lambda$ ( $\lambda$ is the wavelength $)$ are linked by the relation ${ }^{9} r_{C} r_{P}=d^{2} / 4$. The explicit field transformation between the planes $P$ and $C$ is written in Appendix A. An essential point is that, because of the unitarity of this transformation, scalar product (28) defining $\varphi_{p / i}$ can be calculated in any plane. Denoting by $A_{p / i}^{\prime}(r, \varphi, z)$ the image of the mode function $A_{p / i}(r, \varphi, z)$ beyond the lens and using the results given in the appendix, we get

$$
\begin{aligned}
\rho_{p \ell i} \exp \left(-i \varphi_{p \ell i}\right)= & \frac{1}{N^{1 / 2}} \int_{0}^{\infty} r \mathrm{~d} r \int_{0}^{2 \pi} \mathrm{d} \varphi \rho_{P}(r, \varphi) \\
& \times \exp \left(-i \varphi_{P}\right) A_{p \ell i}^{\prime}\left(r, \varphi, z_{P}\right) \\
= & \frac{1}{N^{1 / 2}} \int_{0}^{\infty} r \mathrm{~d} r \int_{0}^{2 \pi} \mathrm{d} \varphi \rho_{P}(r, \varphi) \exp \left(-i \varphi_{P}\right) \\
& \times \exp [-2 i \pi d / \lambda \\
& +(2 p+\ell+1) i \pi / 2] \\
& \times \frac{w_{C}}{w_{P}} A_{p \ell i}\left(r \frac{w_{C}}{w_{P}}, \varphi, z_{C}\right) .
\end{aligned}
$$

Using the reality of $\rho_{P}$ and of the mode functions [see Eqs. (1) and (2)], this equation will ensure that $\varphi_{p / i}=\varphi_{P}$ $+2 \pi d / \lambda-(2 p+\ell+1) \pi / 2$ is a constant modulo $\pi$ for all $p, i$, and even values of $\ell$. Hence the quantity $S_{p / i}$ does not depend on $p, \ell, i$, and we can write

$$
S_{p \ell i}(\omega)=F(\omega) \text { for } 2 p+\ell \text { even. }
$$

As a consequence, expression (25) of the spectrum $S(\omega)$ becomes

$$
S(\omega)=F(\omega) \sum_{p / i}{ }^{\prime} \rho_{p / i}^{2} .
$$

Since by the assumption that the LO field has nonvanishing projection $\rho_{p / i}$ only for $\ell$ even [see Eqs. (28) and (29)], the sum $\Sigma^{\prime}$ can be replaced by the sum over all modes in Eq. (33) and, because $\Sigma_{p \ell i} \rho_{p \ell i}^{2}=1$ by definition, one obtains

$$
S(\omega)=F(\omega),
$$

independent of the intensity configuration $\rho_{P}^{2}(\mathbf{x})$ of the LO field. 

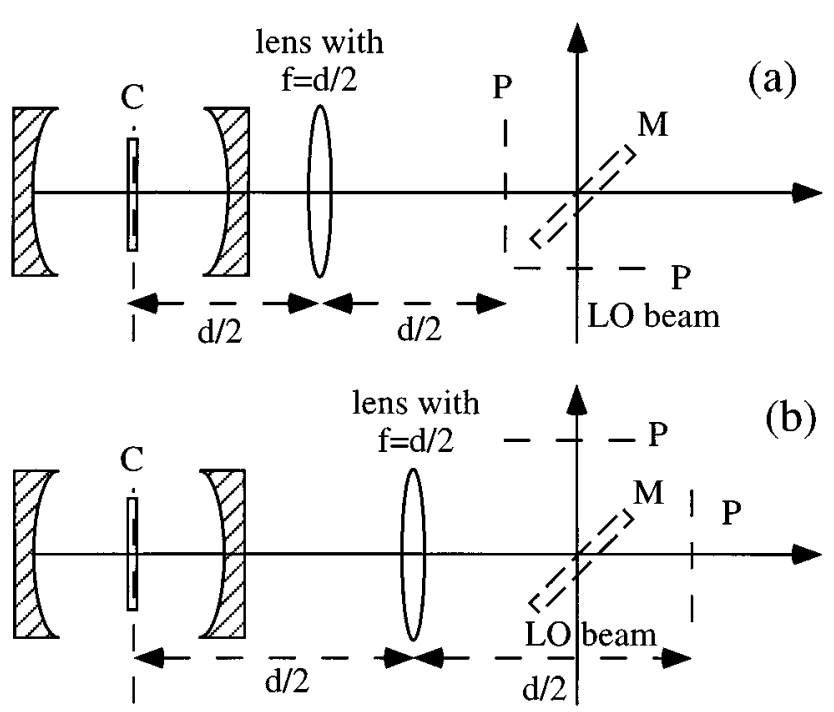

Fig. 3. A lens of focal length $d / 2$ is inserted halfway between the planes $C$ (cavity center) and $P$ (the plane where the LO beam has a uniform phase) that are separated by the distance $d$. Plane $P$ can be either before (case a) or after (case b) the beam splitter. The image of $P$ through the beam splitter is also shown on the figure.

\section{COMPARISON WITH STANDARD MODE MATCHING}

We demonstrated above that if the LO has a flat phase and axial symmetry (but any size) in some plane $P$ at an arbitrary distance $d$ from the cavity center $C$, and if one sets one lens of focal length $f=d / 2$ halfway between $P$ and $C$, then all conditions for noise reduction will be fulfilled. The reason for this is that a lens transforms a beam waist (of any size) in its object focal plane into a beam waist (in general of another size) in its image focal plane in such a way that the flat phase condition is obeyed in both planes.

This is to be compared with standard mode matching, the principle of which is summarized below. In order to mode match squeezed light corresponding to the $\mathrm{TEM}_{00}$ mode of a nonlinear cavity of beam waist $\omega_{a}$ onto a LO of beam waist $\omega_{b}$, both waists being separated by $d$, and the corresponding Rayleigh lengths being $r_{a}=\pi w_{a}^{2} / \lambda$ and $r_{b}=\pi w_{b}^{2} / \lambda$, one has to use a lens of focal length $f$ at a distance $d_{a}$ from $\omega_{a}$, given by

$$
\begin{aligned}
& d_{a}=d \frac{r_{a} \pm \sqrt{r_{a} r_{b}\left[1+\left(r_{a}-r_{b}\right)^{2} / d^{2}\right]}}{\left(r_{a}-r_{b}\right)} \\
& d_{b}=d \frac{r_{b} \pm \sqrt{r_{a} r_{b}\left[1+\left(r_{a}-r_{b}\right)^{2} / d^{2}\right]}}{\left(r_{b}-r_{a}\right)}=d-d_{a} \\
& f=\frac{\left(d_{a}^{2}+r_{a}^{2}\right)\left(d_{b}^{2}+r_{b}^{2}\right)}{\left(d_{a}^{2} d_{b}+d_{a} d_{b}^{2}+d_{b} r_{a}^{2}+d_{a} r_{b}^{2}\right)} .
\end{aligned}
$$

Therefore not only the position but also the size of the beam waist of the LO have to be used to determine the focal length and the position of the lens. Let us remark that if one chooses to put the lens at the midpoint between the two waists, $\left(d_{a}=d_{b}=d / 2\right)$, two cases are interesting:
- One has $r_{a}=r_{b}$ and $f=d / 4+r_{a}^{2} / d$. This corresponds to a limit of the equations above (which look singular for $r_{a}=r_{b}$, but actually are not), obtained in the case where the cavity and the LO beam waists have the same size (the value of $f$ still depends on that size).

- Or one has $f=d / 2$ and $r_{a}=d^{2} /\left(4 r_{b}\right)$. This is what we use here, by changing a waist into a waist of another size. In general, this would destroy the modematching condition, but it does not matter in our case: Only the flat phase condition is important.

Let us remark that several lenses of focal length $f_{j}$ can be used instead of one provided that $2 \Sigma_{j} f_{j}=d$ and that the image focal plane of one lens coincides with the object focal plane of the next one. One can therefore expect a significant simplification of the mode-matching process by using the procedure described in this paper.

\section{CONCLUSIONS AND CRITICAL COMMENTS}

In order to observe the level of squeezing in the squeezed vacuum state emitted by a degenerate OPO below threshold, it is easy to use a LO field of Gaussian shape. However, in order to match the Gaussian with the fundamental mode of the OPO cavity, one must control the phase curvature of the Gaussian LO and its width. The required mode-matching procedure is usually not straightforward, and the possible squeezing available in modes other than $\mathrm{TEM}_{00}$ remains irrelevant. The analysis in this paper has shown that, by using a confocal cavity and a matching lens independent of the LO size, a Gaussian LO of arbitrary width allows one to use the full available degree of squeezing. In addition, instead of a Gaussian, one can consider any other intensity configuration $f^{2}(r, \varphi)$, provided that $f(r, \varphi+\pi)=f(r, \varphi)$; for example, any axially symmetrical configuration is good.

All the previous analysis remains unchanged if we assume that the signal-field frequency $\omega_{s}$ is close to resonance with the modes with $\ell$ odd instead of $\ell$ even. In this case, in all considerations concerning the signal field one must simply replace the specification $\ell$ even by $\ell$ odd and vice versa.

From a practical viewpoint, in the previous analysis we must assume that Gauss-Laguerre modes of high order should not be relevant in expansion (4), which means also that the LO beam waist should be neither too small nor too large. This requirement is linked to the following:

(1) The plane-wave approximation for the pump beam, which cannot be fulfilled with an arbitrary accuracy.

(2) The finite angular width of the phase-matching condition in the nonlinear crystal.

(3) The spherical aberrations, which will lift the degeneracy of higher-order modes if simple spherical mirrors are used. This effect, as well as the one of phase mismatch in the nonlinear crystal, can in principle be circumvented by an appropriate design (e.g., using aspheric mirrors), but makes it difficult to give a simple estimate of the number of squeezed modes. 
(4) Possible slight deviations from exact confocality, which would imply that modes with different $2 p+\ell$ have slightly different frequencies, and the frequency difference from the fundamental mode would become large in the case of high order modes. As is shown in Ref. 8, near the threshold of the OPO the level of squeezing for $\phi_{L}=\pi / 2$ and $\omega=0$ becomes very sensitive to the detuning $\Delta$, in the sense that there is large squeezing in a narrow range of $\Delta$ and no squeezing at all out of this range. One should therefore stay somewhat away from threshold, where precise confocality becomes very critical, even if this reduces the maximum squeezing level attainable.

To conclude, let us summarize the circumstances that allowed us to relax strongly the usual mode-matching conditions, so that the observed degree of squeezing becomes widely independent on the spatial configuration of the LO field:

(i) The use of a confocal cavity, which is a stable cavity in which all Gauss-Laguerre modes with a given parity of $\ell$ are frequency degenerate;

(ii) The use of a thin crystal at the cavity center, excited with a nearly plane-wave pump, which allows the spectrum of squeezing to be given by a superposition of uncorrelated single-mode contributions;

(iii) The use of a LO field with the axial symmetry $f(r, \varphi+\pi)=f(r, \varphi)$, in a configuration such that there is a plane orthogonal to the direction of propagation where the LO has a uniform phase modulo $\pi$ (beam waist);

(iv) The use of a matching lens from the cavity to the LO beam waists, which makes all phases $\varphi_{p / i}$ remain equal to one another in the propagation outside the cavity and therefore ensures that all modes will contribute to maximum noise reduction.

Finally, we note that the condition (ii) does not hold in the general case of squeezed fields emitted by nonlinear cavities, where the nonlinear coupling also creates correlations between the transverse modes. However, possible generalizations of the techniques introduced here to more complex situations are certainly worth investigating. Although they do not solve all problems involved in the production and the use of squeezed light, detailed consideration and control of the spatial aspects of squeezing can certainly help towards the production of reliable highly squeezed light. ${ }^{12}$

\section{APPENDIX A: PROPAGATION OF THE GAUSS-LAGUERRE MODES}

The general expression of Gauss-Laguerre modes ${ }^{9}$ at point $z$ along the beam is

$$
A_{p 0}(r, \varphi, z)=\frac{2}{w(z) \sqrt{2 \pi}} L_{p}^{0}\left[\frac{2 r^{2}}{w(z)^{2}}\right] \exp \left[-\frac{r^{2}}{w(z)^{2}}\right]
$$

$$
\begin{aligned}
A_{p \ell i}(r, \varphi, z)= & \frac{2}{w(z) \sqrt{\pi}}\left[\frac{p !}{(p+\ell) !}\right]^{1 / 2}\left[\frac{2 r^{2}}{w(z)^{2}}\right]^{\ell / 2} \\
& \times L_{p}^{\prime}\left[\frac{2 r^{2}}{w(z)^{2}}\right] \exp \left[-\frac{r^{2}}{w(z)^{2}}\right] \\
& \times \exp \left[-2 i \pi \frac{r^{2}}{2 \lambda R(z)}\right. \\
& \left.+i(p+2 \ell+1) \tan ^{-1}\left(\frac{z}{r_{C}}\right)\right] \\
& \times \begin{cases}\cos (\ell \varphi) \quad \text { for } i=1 \\
\sin (\ell \varphi) \quad \text { for } i=2\end{cases}
\end{aligned}
$$

where $w(z)$ and $R(z)$ are the beam waist and the radius of curvature at point $z$, and $r_{C}=\pi w_{C}^{2} / \lambda$ is the Rayleigh length. ${ }^{9}$ Propagation from plane $C$ to the lens at distance $d / 2$ will therefore introduce the shift $\phi_{C}=-\pi d / \lambda$ $+(2 p+\ell+1) \tan ^{-1}\left[d /\left(2 r_{C}\right)\right]$ whereas from the lens to plane $P$ the shift is $\phi_{P}=-\pi d / \lambda+(2 p+\ell$ $+1) \tan ^{-1}\left[d /\left(2 r_{P}\right)\right]$. The total phase shift is therefore

$$
\begin{aligned}
\phi_{C}+\phi_{P}= & -2 \pi d / \lambda+(2 p+\ell+1) \\
& \times\left\{\tan ^{-1}\left[d /\left(2 r_{C}\right)\right]+\tan ^{-1}\left[d /\left(2 r_{P}\right)\right]\right\} \\
= & -2 \pi d / \lambda+(2 p+\ell+1) \\
& \times\left\{\tan ^{-1}\left[d /\left(2 r_{C}\right)\right]+\tan ^{-1}\left(2 r_{C} / d\right)\right\} \\
= & -2 \pi d / \lambda+(2 p+\ell+1) \pi / 2,
\end{aligned}
$$

where we use the relation ${ }^{9} r_{C} r_{P}=d^{2} / 4$, which describes the changes in the wave fronts that are due to the lens (the constant phase shift that is due to the lens is irrelevant and has been omitted here). As a consequence, the lens induces the following transformation: If $F_{C}(\mathbf{x})$ is the field configuration in the beam waist plane $C$, and if one has the expansion

$$
F_{C}(\mathbf{x})=\sum_{p / i} f_{p / i} A_{p / i}(r, \varphi, 0),
$$

where $r=\left(x^{2}+y^{2}\right)^{1 / 2}$, then the corresponding field in the other beam-waist plane $P$ will be

$$
\begin{aligned}
F_{P}(\mathbf{x})= & \sum_{p \ell i} f_{p \ell i} A_{p \ell i}^{\prime}\left(r, \varphi, z_{P}\right) \\
= & \sum_{p \ell i} f_{p \ell i} \exp \left[-\frac{2 i \pi d}{\lambda}+(2 p+\ell+1) \frac{i \pi}{2}\right] \\
& \times \frac{w_{C}}{w_{P}} A_{p \ell i}\left(r \frac{w_{C}}{w_{P}}, \varphi, 0\right),
\end{aligned}
$$

where $A_{p \ell i}^{\prime}\left(r, \varphi, z_{P}\right)$ denote the modes functions in plane $P$. The same result can also be obtained using $A B C D$ matrices (see, e.g., Ref. 9, Section 20.2).

\section{ACKNOWLEDGMENTS}

We are grateful to A. Bramati for a helpful discussion. This research has been carried out in the framework of the European Strategic Program for Information Technol- 
ogy Basic Research Project 6934 (Quantum Information Technology) and the Human Capital and Mobility network Nonclassical Light.

\section{REFERENCES}

1. H. P. Yuen, "Two-photon coherent states of the radiation field," Phys. Rev. A 13, 2226-2243 (1976).

2. M. I. Kolobov and I. V. Sokolov, "Spatial behaviour of squeezed states of light and quantum noise in optical images," Sov. Phys. JETP 69, 1097-1105 (1989).

3. M. I. Kolobov and I. V. Sokolov, "Squeezed states of light and quantum noise-free optical images," Phys. Lett. A 140, 101-103 (1989).

4. M. I. Kolobov and I. V. Sokolov, "Multimode squeezing, antibunching in space and noise-free optical images," Europhys. Lett. 15, 271-274 (1991).

5. A. La Porta and R. E. Slusher, "Squeezing limits at high parametric gain,” Phys. Rev. A 44, 2013-2025 (1991).
6. C. Kim and P. Kumar, "Quadrature-squeezed light detection using a self-generated matched local oscillator," Phys. Rev. Lett. 73, 1605-1609 (1994).

7. L. A. Lugiato and A. Gatti, "Spatial structure of a squeezed vacuum," Phys. Rev. Lett. 70, 3868-3871 (1993).

8. A. Gatti and L. A. Lugiato, "Quantum images and critical fluctuations in the optical parametric oscillator below threshold," Phys. Rev. A 52, 1675-1690 (1995).

9. A. E. Siegman, Lasers (University Science, Mill Valley, Calif., 1986).

10. M. J. Collett and D. F. Walls, "Squeezing spectra for non-linear optical systems," Phys. Rev. A 32, 2887-2892 (1985).

11. C. M. Savage and D. F. Walls, "Squeezing by parametric oscillation and intracavity four-wave mixing," J. Opt. Soc. Am. B 4, 1514-1519 (1987).

12. K. Schneider, R. Bruckmeier, H. Hansen, S. Schiller, and J. Mlynek, "Bright squeezed light generation by a continuouswave semi-monolithic parametric amplifier," Opt. Lett. 21, 1396-1398 (1996). 